\title{
maquinaria y aparatos para la construcción en invierno
}

\author{
Dipl. Ing. Eugen SCHLEICHER, Frankfurt.
}

Conferencia pronunciada en el Centro Téenico de Essen (Alemanja)

Una obra que debe estar dispuesta para trabajar en invierno, tiene que cumplir con los requisitos correspondientes, disponer de los medios de trabajo baj̧o estas condioiones $y$ adaptarse el proyecto de realizacion para esta época del año.

No sólo se trata de los métodos constructivos que demuestran un cierto rendimiento, sino que se deben considerar también las indicaciones especiales en la realización y dispositivos de la obra Las Asociaciones Alemanas de la construcción en la República Federal han podido comprobar un aumento considerable en las actividades de la construeción durante el último invierno, confirmándose que el 90 por 100 de las obras realizadas desde el 1-11-60 al 28-2-61, por un valor de unos 300.000 .000 D.M., no han tenido dificultad alguna en cuanto a los trabajos sometidos a las condiciones del invierno. Hemos de tener en cuenta que ula construcción de inviernow se ha realizado a temperaturas de -7 a $-10^{\circ} \mathrm{C}$, siendo esto un exito gracias a las medidas que se tomaron en las obras. Hay que considerar que estas medidas tenian que garantizar el desarrollo de los trabajos con frío y con lluvia. Esto es un resultado asombroso e inesperado después de haber tratado hace muchos años en a República Federal la construcción en invierno en gran escala, pudiéndose decir hoy dia, por los muchos ejemplos de obras realizadas, que esto es factible.

\section{Trabajos preliminares}

La obra de invierno exige a las empresas constructoras ciertos requisitos, que se pueden cumplir sin. grandes dificultades dado el estado actual de la técnica del trabajo en cualquier obra bien organizada. La falta de consideracion de estos requisitos preliminares puede conducir a una interrupeión de los trabajos o a perjuicios téenjeos. con estas medidas tomadas para la construcción en invierno se consigue un amplio éxito económico, éxito que se basa en una preparación de los dispositivos en la obra relacionada indirectamente con el desarrollo de la misma con el tiempo. En primer término, es importante la elección del método constructivo adecuado, y de la maquinaria y aparatos para cumplir con el rendimiento exigido.

En la elección đe los hormigones es natural este requisito. Nadie tratará de emplear una máquina para producir sólo 250 l/earga si precisa un volumen de hormigón de 8 a $10 \mathrm{~m}^{3} / \mathrm{hora}$. En la obra de invierno es preciso disponer de una caldera de agua caliente con una capacidad que corresponda con el volumen de agua necesario. Si la capacidad es reducida origina paradas en el trabajo, y si hay un exceso ocasiona un gasto inútil.

La maquinaria a emplear en los trabajos de invierno, se puede dimensionar de la misma forma que el resto de los elementos: mezcladoras, elevadores, grúas, cintas transportadoras, etc., que deben trabajar, como es natural, con un cierto margen de tolerancia. Todo esto se basa en los conocimientos simples de física y quimica relacionados con el comportamiento de los materiales a baja temperatura que determinan el desarrollo de la obra. Serla interesante tratar de] aspecto cuantitativo de los dispositivos para una construcción de invierno, pero esto no lo podemos tratar en este articulo. En esta ocasión, sólo nos vemos obligados a referirnos a la maquinaria empleada normalmente en la construcción de invierno. Se comprende que el trabajo de hormigonado represente la mayor dificultad en invierno, siendo una especialidad que no deja de tener interés.

Fl material hormigón se compone de árido, cemento y agua, en los casos normales, Este material endurece según un proceso químico con el tiempo. La intensidad de este proceso depende de la tomperatura de este conjunto y del ambiente en que tiene lugar. 
Todas las máquinas y aparatos empleados en la construcción en invierno-se trata del hormigonado-se refieren a la producción de agua caliente para conseguir el estado térmico adecuado en los materiales que han sufrido un enfriamiento.

\section{Ceneradores de calor}

Los diferentes componentes del hormigón no se eneuentran en invierno con temperatura suficiente como para conseguir un endurecimiento normal, tal como octurre a temperaturas de 18 a $20^{\circ} \mathrm{C}$. La arena, la grava, el agua y el cemento se deben calentar independientemente con objeto de conseguir que tenga el hormigon fresco una temperatura injeial suficiente para que pueda tener lugar el endurecimiento. Se tiene que mantener a esta temperatura un determinado tiempo hasta conseguir una cierta resistencia. Como soporte de calor se dispone de agua caliente, vapor (a baja o alta presión), aire caliente, calor por rayos infrarrojos y calor por resistencia eléctrica. Para poder hacerse una idea de la cantidad de calor consumido-con esto se puede obtener la capacidad de los generadores de calor y las medidas de aisiamiento térmico en obre-w, el sorganizador del trabajo» ha de considerar el rendimiento exigido por dia y los limites de temperatura. Este debe de conocer hasta qué temperatura minima es posible hormigonar, pues esta indicación es interesante en el indice de rendimiento.

Para estas temperaturas mínimas hay que disponer de instalaciones de calentamiento y medidas de protección, independientemente de que estas temperaturas lleguen o no a producirse. Por ejempo, si se tiene que prever un hormigonado hasta $-10^{\circ} \mathrm{C}$ se necesita disponer de calor suficiente para contrarrestar esta temperatura exterior. A base de este dato inicial es posible calcular la cantidad de calor necesaria para el rendimiento previsto por hora. La temperatura inicial de los áridos se debe considerar igual a la temperatura exterior, a no ser que se haya tomado alguna medida especial de protección. La del agua se conoce también; cuando se obtiene de una tubería en invierno se puede considerar de 5 a $6^{\circ} \mathrm{C}$. Como pérdidas de temperatura en el transporte de la hormigonera al lugar de colocación y la pérdida en este lugar se consideran los valores de la experiencia.

La temperatura necesaria en la colocación del bormigón fija los limites para la determinación del calor necesario, Por ejemplo, para una hormigoner: de 500 litros se precisa, bajo condiciones normales para hormigonar hasta $-10^{\circ} \mathrm{C}$, una inversión de calor de 120.000 a $130.000 \mathrm{kcal} / \mathrm{hora}$, lo que representa unos $200 \mathrm{~kg}$ de vapor a baja presión por hora.

Este consumo de calor es necesario calcularlo para cada instalación especial de la obra. Con estos valores de base se puede elegir, en cada caso, la economia y la efectividad técnica de las máquinas, calderas de vapor, instalaciones ce agua caliente, radiadores o generadores de agua caliente.

Antes de mencionar con detalle los diferentes tipos de máquinas, queremos dar a conocer otras propiedades que precisan tener todas las máquinas empleadas en la obra de invierno:

1. El calor se precisa, frecuentemente, en lugares de dificil acceso. Por ejemplo, es necesario liberar a los encolrados y armaduras de la nieve o hielo que les cubren; entonces, se debe poder efectuar un acceso de calor sin instalación complicada.

2. Se tiene que poder suministrar una cantidad constante de calor en un plazo largo; por ejemplo, durante varias horas.

3. La fuente de calor se debe poder poner rápidamente y sin grandes preparativos a pleno rendimiento y poder desconectar sin pérdidas.

4. La producción de calor se debe poder regular entre ciertos límites.

5. Hay que tener en euenta que los obreros de la construcción no son mecánicos. En consecuencia, los aparatos deben trabajar con una conservación reducida, no ser de hechura complícada $y$, por tanto, han de ser de tácil manejo.

6. El combustible ha de ser lo más barato posible $\mathrm{y}$ tener un rendimiento de calor especificamente elevado para que con poco peso se pueda calentar un plazo largo. Este combustible ha de ser insensible al frio considerando nuestra gama de temperaturas.

7. Los pesos de los aparatos han de ser reducidos, con objeto de poderlos trasladar con facilidad en la obra o a otros lugares de trabajo.

8. Los aparatos sin corriente tienen ventaja en muchas obras pequeñas de edificación donde es difícil la conexión a la red, especialmente si se trata de un consumo elevado.

9. Los aparatos de calefacción deben servir para múltiples fines de calentamiento en la obra.

10. Los aparatos han de ser económicos, con una conservación reducida, ya que tienen que trabajar todo el invierno en la obra sometidos a las inclemencias atmosféricas y sin trabajar parte del tiempo. 


\section{La instalación de agua caliente}

Con frecuencia es suficiente dar al hormigón el calor necesario a base de calentar el agua de amasado. En el caso de temperaturas ambiente no muy bajas, es decir, en temperaturas en áridos $\mathrm{y}$ aglomerante no muy reducidas, la temperatura necesaria para la puesta en obra del hormigón se consigue empleando un agua de amasado calentada de 60 a $80^{\circ} \mathrm{C}$.

Esto es muy sencillo de calcular, ya que el agua de amasado es buen transmisor de calor como consecuencia del elevado calor especifico del agua. El calor especifico del agua es 1 , es decir, $1 \mathrm{~kg}$ de agua recoge, por grado de temperatura, $1 \mathrm{kcal}$ al calentarse y al enfriarse lo cede en igual'proporción. La arena, la grava y, también, el cemento, tienen un calor especifico mucho más bajo $(\sim 0,2)$. Esto quiere decir que al calentarse estos materiales, aun con poco calor, consiguen una temperatura elevada y, a la inversa, al ceder calor; o sea, al mezclarse con otras partes frías del hormigón fresco se liberan pequeñas cantidades de calor.

El calentamiento del hormigón por el agua de amasado presenta otras ventajas:

- El calentamiento de esta agua se puede conseguir fácilmente en cualquier condición de obra. Los gastos de salarios para este trabajo son reducidos.

- El agua al calentarse lo hace uniformemente.

- Con la ayuda de instrumentos simples se controla fácilmente la temperatura conseguida.

- Al efectuarse el intercambio de calor entre el agua caliente y los áridos o los aglomerantes, la transmisión del calor a la masa más fría tiene lugar con rapidez y uniformidad.

- Además, en cualquier obra de invierno se precisa agua caliente para otros fines.

Todos estos puntos de vista son los motivos que originan que en la mayoría de las obras y, sobre todo, en las pequeñas, se emplea el agua como soporte principal, a veces como único, para transmitir calor.

Solamente en algunas obras pequeñas podemos ver todavía hoy calderas o serpentines que utilizan combustible sólido en forma de madera, carbón o cok (fig. 1). Esta forma de calentar el agua es incómoda para grandes cantidades y, además, antieconómica. Por este motivo se han construido una serie de aparatos que trabajan con un rendimiento superior. Por ejemplo, existen serpentines de caldeo en un horno circular calentado por un generador de aceite que se denomina "Schwing-FenerGerät» (posteriormente nos referiremos de nuevo a este aparato que aparece en la figura 2). El serpentín de caldeo se conecta a la tubería de agua, de forma que se dispone de un calentador continuo, simple y efectivo. Este horno o estufa es ligero, sin complicaciones y con una estructura muy sencilla, suministrando en pocos minutos una fuente continua de agua caliente.
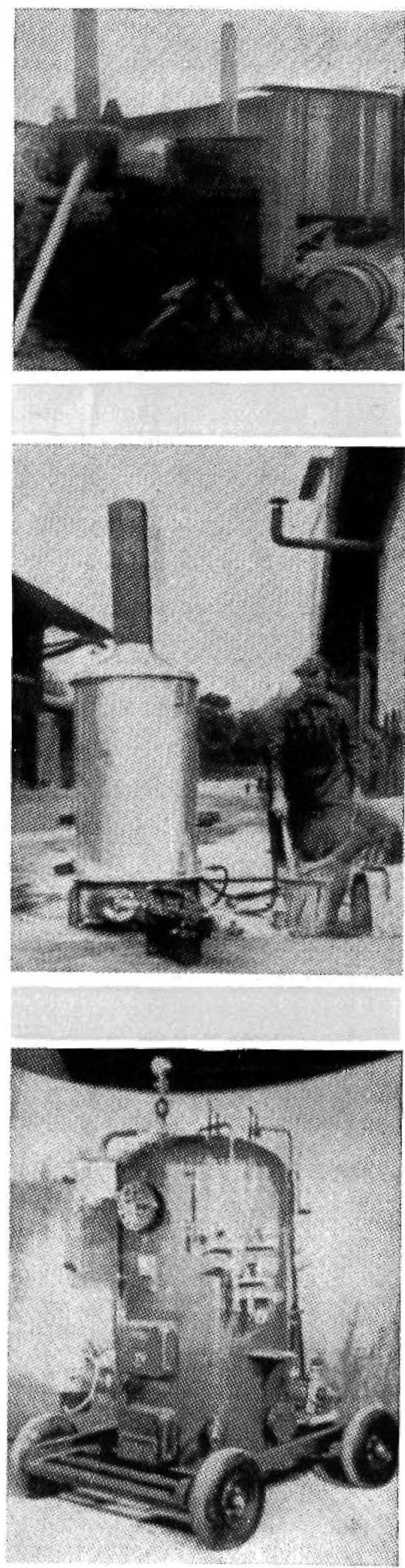

Fig. 1. Caldera fija para la preparación de agua para la elaboracíón de hormigón. Fig. 2. Estufa circular, en la que se encuentra un serpentin para la preparación de agua. Ruemador: aparato de llama derna con dispositivo móvil. 
Como el quemador trabaja automáticamente un periodo de tiempo largo, esta máquina requiere poca conservación. La temperatura del agua se puede regular fácilmente abriendo o cerrando más la llave de paso. Esta máquina proporciona agua suficiente para una amasadora de tipo medio. si, por cualquier motivo, se precisa vapor de este serpentín, se consigue fácilmente cerrando más el paso del agua,

Este dispositivo corresponde en su funcionamiento al serpentín simple, pero tiene la ventaja de que al trabajar con madera o carbón se obtiene mayor efectividad y un largo periodo de calentamiento. También se emplean calentadores eléctricos de paso continuo, en casos especiales según las exigencias de la obra. Una fábrica grande alemana construye aparatos de este tipo con un consumo de 12 a $96 \mathrm{~kW}$, pero tienen la desventaja de que en la obra no se consume generalmente esta capacidad continuamente para poder conseguir un funcionamiento económico del aparato.

El agua caliente se puede introducir a la hormigonera (a la mezcla seca) por medio de un depósito intermediario.

Desde hace varios años se utilizan también relojes de agua para alimentar el agua de amasado directamente a las hormigoneras.

Existen calderas para agua caliente que satisfacen todas las exigencias modernas desde el punto de vista constructivo (fig. 3). Citaremos un modelo que produce agua caliente para una amasadora de 375 o 500 litros con garantias de funcionamiento.

Existen, además, aparatos combinados que suministran agua caliente, vapor y aire caliente.

En el caso de temperaturas bajas $\mathrm{y}$, frecuentemente, ya a $0^{\circ} \mathrm{C}$, no es suficiente la cantidad de calor transmitida por el agua de amasado para el hormigón, especialmente cuando hace mucho aire.

Ya se ha indicado anteriormente que cada $\mathrm{kg}$ de agua calentada a $1^{\circ} \mathrm{C}$ acumula $1 \mathrm{kcal}$. Un $\mathrm{kg}$ de agua calentada a $60^{\circ} \mathrm{C}$ y que se enfria a $20^{\circ} \mathrm{C}$, al ponerse en contacto con la mezcla fresca de hormigón solamente dispone de $40 \mathrm{kcal}$.

\section{Generadores de vapor para la construcción en invierno}

Cuando se precisan unas cantidades de calor mayores, es necesario emplear vapor. Este es un soporté de calor que cede muchas calorías por unidad de peso; el vapor de agua al condensar cede, aproximadamente, $536 \mathrm{kcal} / \mathrm{kg}$. Esto indica, naturalmerte, que es preciso emplear la misma cantidad de calor al pasar agua líquida a $100^{\circ} \mathrm{C}$ en su forma de vapor.

El vapor presente, por otra parte, la ventaja de transportarse fácilmente a distancias superiores sin pérdidas importantes de calor, aun a falta de un buen aislamiento de la conducción.

Por una parte, el vapor se puede utjlizar para calentar el agua de amasado. Para ello se precisa una bateria de mezcla o un dispositỉvo determinado con objeto de conseguir la cesión total del calor del vapor al agua.

También se pueden-que es la solución corriente-calentar los áridos directamente soplando vapor caliente (fig. 4); es una solución muy corriente. Esto se realiza generalmente con lanzas de vapor formadas por tubos perforados de 1 a $1 \frac{1 / 2}{2}$ pulgadas que se introducen dentro del montón del árido. La cesión de calor por este método centra su importancia en la condensación de vapor; el agua caliente formada crea un aumento en el contenido de humedad de los áridos. Este es un punto importante a considerar en la obra. El calentamiento con vapor crea siempre una fuente de agua que puede originar posiblemente dificultades en el desarrollo de la obra, ya que esta parte de agua puede helarse.

Si se utiliza vapor para eliminar la nieve de los encofrados o de las armaduras, se humedecen estos elementos (flg. 5). Es importante que después de esta fase previa del trabajo se hormigone inmediatamente para evitar que la humedad originada pueda helarse.

Toda formación de hielo en la obra de invierno es desagradable. Si, por ejemplo, se hielan los aridos 0 se tiene que retirar hielo de otras partes tales como encofrados o armaduras, hay que invertir gran cantidad de calor sólo para el deshielo. Un kilo de hielo a $0^{\circ} \mathrm{C}$ precisa, para su transformación en agua a $0^{\circ} \mathrm{C}$ (sólo para fundirlo), $80 \mathrm{kcal}$.

Si nos hacemos la idea de que con un contenido del 5 por 100 de humedad a los áridos-porcentaje normal en invierno-debemos considerar un contenido de unos 250 litros de agua, se precisan sólo para su deshielo unas 20.000 kcal. Por el contrario, al tratarse de los áridos secos, para calentarlos de 0 a $20^{\circ} \mathrm{C}$ se precisan sólo $5.200 \mathrm{kcal}$. El cemento precisa, por su parte, para este aumento de temperatura unas $1.000 \mathrm{kcal}$. Estos valores se refieren a $1 \mathrm{~m}^{3}$ de hormigón. Puede apreciarse la gran inversión suplementaria motivada por el agua embebida en lo poros del árido cuando está helado. 
Al introducir vapor en los áridos con ayuda de una lanza, se debe calentar solamente la cantidad que se va a emplear inmediatamente, y prestar especial atención a que no se alimente o introduzca demasiado vapor. Aparte de una pérdida innecesaria de vapor si se alimenta mucho, se condensa demasiada agua.

Empleando vapor se consiguen temperaturas elevadas en los áridos. Estos captan el calor en forma de cilindro alrededor de la lanza y el calentamiento sucede, en parte, directamente por conducción $\mathrm{y}$, en parte, por convección. Como en un tiempo relativamente corto se reduce mucho la conducción directa de calor, es aconsejable cambiar la situación de la lanza con mucha frecuencia. La cubrición del árido con una lona puede evitar pérdidas de vapor.

En las obras de la República Federal se suelen usar como generadores de calor los de baja presión. También se ha propuesto emplear vapor a alta presión (hasta 12 atm. o más) para conseguir mayor cantidad de calor y una cantidad más reducida de agua condensada. Ya con un cálculo simple se demuestra que esta suposición sólo es acertada en parte. Vapor saturado a $0,51 \mathrm{~atm}$., por consiguiente vapor a baja presión, suministra, aproximadamente, unas $590 \mathrm{kcal} / \mathrm{kg}$ de vapor. El vapor a 8 atm (alta presión) lleva $612 \mathrm{kcal} / \mathrm{kg}$ de vapor. Un vapor saturado a 3 atm y recalentado a $300^{\circ} \mathrm{C}$ suministra $682 \mathrm{kcal} / \mathrm{kg}$ de agua.

El vapor a alta presión no tiene un interés especial más que en aquellos lugares donde son necesarias conducciones a distancias mayores o donde se emplean tuberías de poca sección. En consecuencia, no vale la pena disponer instalaciones de alta presión en obras normales sólo con la idea de conseguir una posible reducción en la condensación del agua.

En Alemania se construyen ya calderas móviles para vapor a alta presión que, en cierto modo, son muy útiles. En determinadas condiciones, en una obra amplia donde se precisan grandes cantidades de calor, podría ser interesante una instalación de este tipo, análoga a las empleadas en Suecia.

La instalación de alta presión va montada sobre un carro, siendo manejada

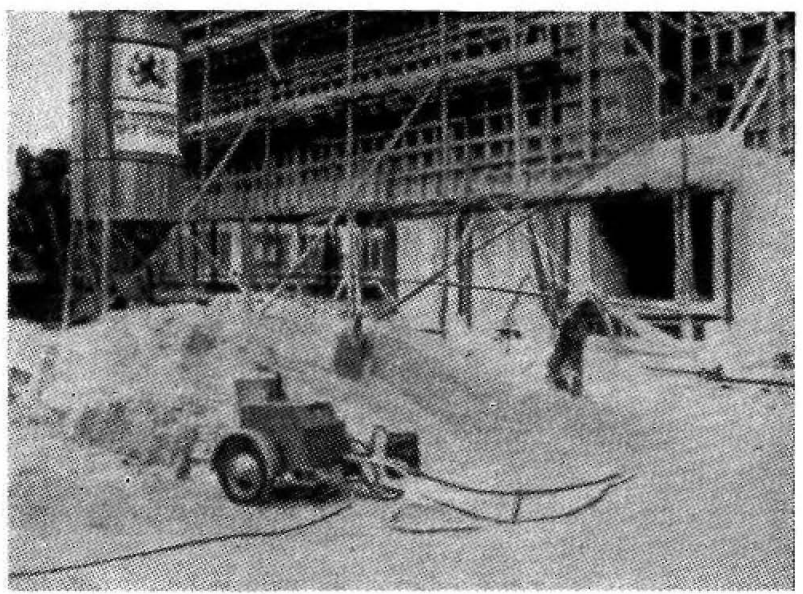

Fig. 4. - Calentados por lanzas de. vapor.

Fig. 5. - Limpieza de nieve en las armaduras con ayuda de vapor.

Fig. 6. - Instalación móvil para la preparacion de vapor para la insen obras de im. portancia.
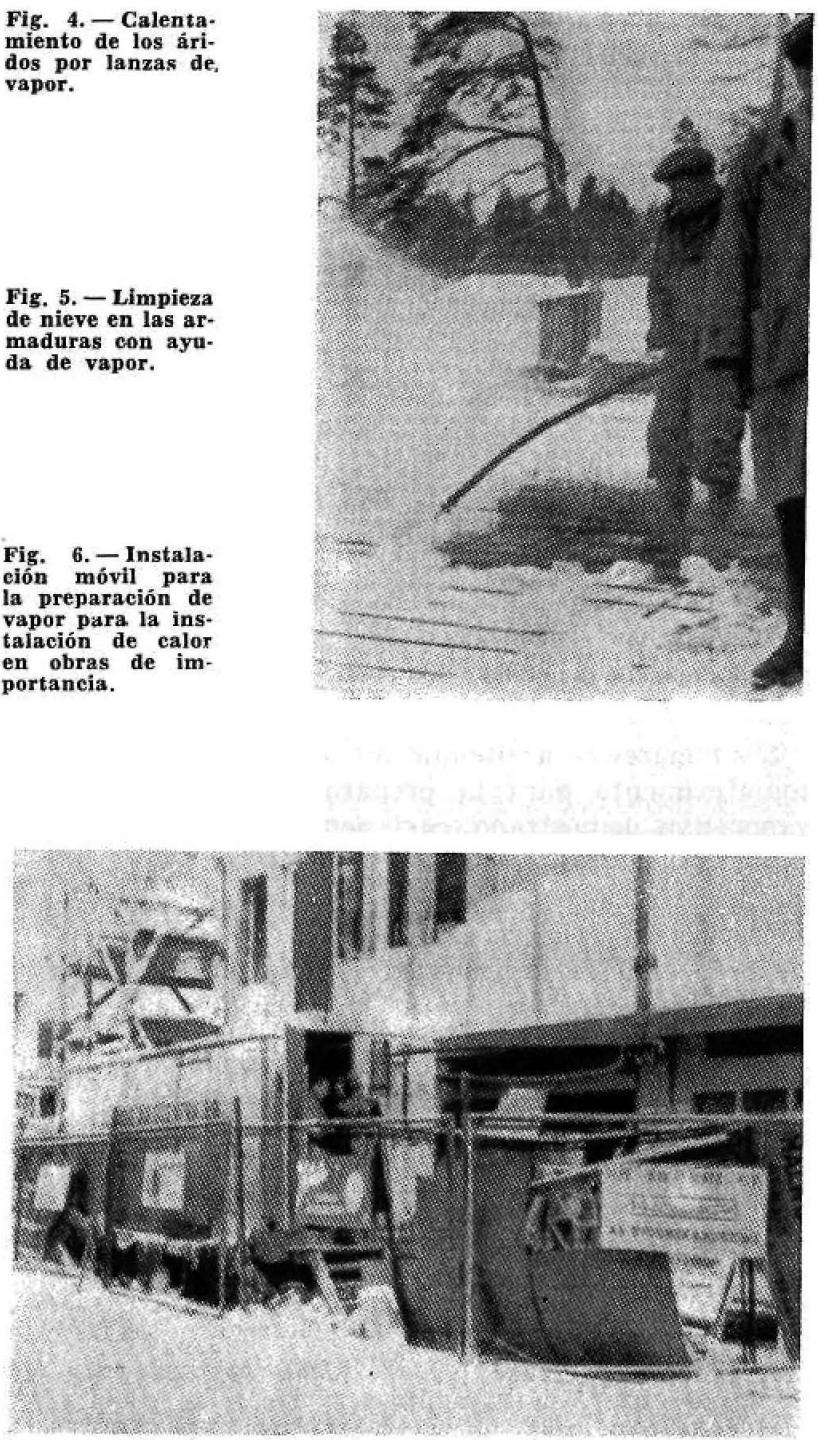
por un operario especializado que trabaja para la empresa constructora y realiza, a la vez, todas las instalaciones técnicotérmicas para la obra del invierno. Las cantidades de calor a suministrar se garantizan por contrato (fig. 6).

Las calderas de baja presión trabajan a presiones de 0,5 a 1 atm. En los últimos años han aparecido en el mercado calderas calentadas por aceite que resultan económicas por su precio. Su rendimiento de calor por hora es, según la amplitud de la superficie de calentamiento, de 150 a $200 \mathrm{~kg} / \mathrm{h}$ ora que se producen en el plazo kreve de calentamiento (figura 7). Su volumen térmico corresponde acertadamente con el tipo de preparación de calor necesario en gran número de obras pequeñas y de tipo medio. Algunos tipos de estas instalaciones de baja presión poseen, junto al generador de calor, un generador de agua caliente (figura 8).

Las instalaciones de alta presión-como ya hemos indicado anteriormenteno se hallan casi en las obras alemanas, ya que su funcionamiento encuentra dificultades en relación con las disposiciones fijadas; se emplean en el extranjero en gran escala. Estas trabajan hasta unas presiones de vapor de $12 \mathrm{~atm}$. El autor considera que en Alemania aparecerán pocas obras en el futuro que pudiesen conseguir ventajas con estas instalaciones. Su instalación difícil y su servicio son inadecuados para las obras de tipo pequeño y medio.

Los hogares de aceite que trabajan automáticamente para la preparación de vapor han demostrado, casi siempre, su economia como fuente de calor frente a los hogares con combustibles sólidos. La conservación y la manipulación es más fácil, lo cual es importante, porque en las obras debe ahorrarse el mayor número posible de operarios especializados. Como ejemplo de un generador adecuado y moderno para una obra de invierno describiremos brevemente el generador Kärcher (figura 9). Pesa, aproximadamente, $350 \mathrm{~kg}$ y es móvil. Es regulable y puede suministrar hasta $280 \mathrm{~kg}$ de vapor por hora. A elección del usuario puede trabajar como instalación de baja presión o con presión de vapor hasta de $10 \mathrm{~atm}$. A baja temperatura consigue también plena capacidad en pocos

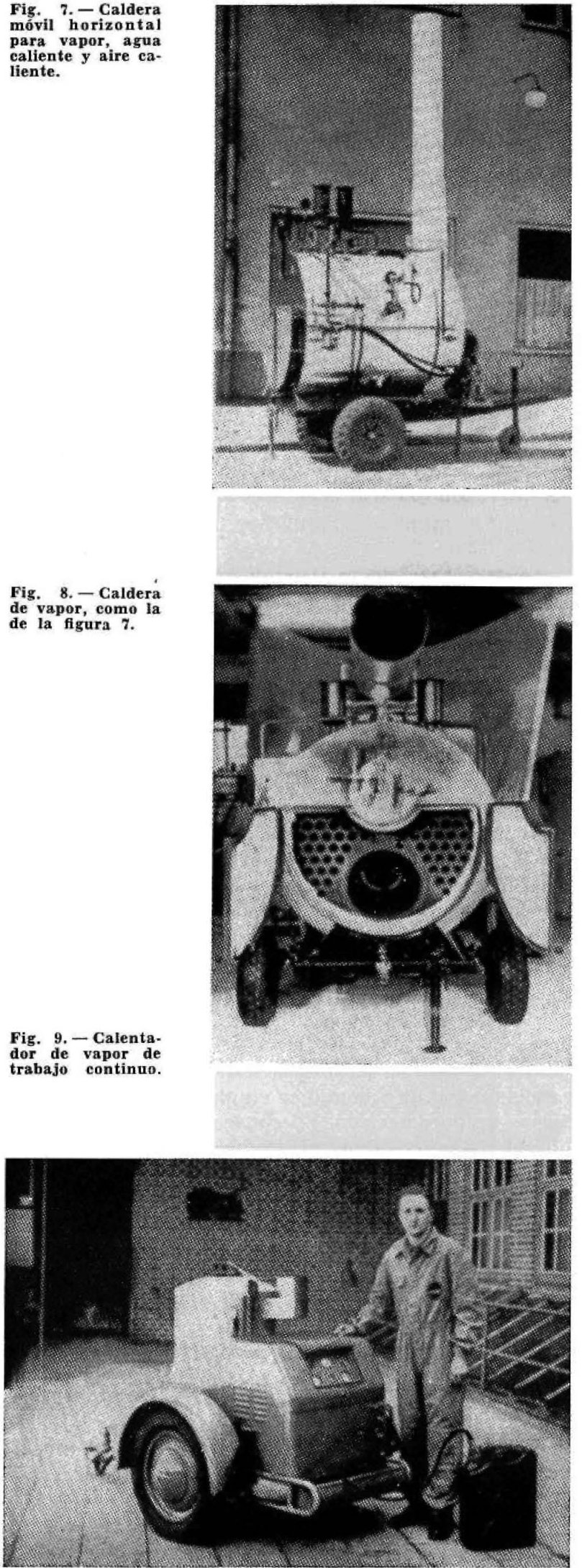


minutos de calentamiento. La cantidad de calor suministrada puede ser hasta de $180.000 \mathrm{kcal} /$ hora con una temperatura máxima del vapor de $184^{\circ} \mathrm{C}$. Este aparato tiene la ventaja de no encontrarse sometido al control legal de las calderas de paso obligado. Como dispositivos de seguridad dispone de una válvula, de un dispositivo para indicar el defecto del agua, de un conmutador de sobrepresión y de un termostato. El generador de calor de este aparato se compone de intercambiador y del grupo de bombeo. El encendido es eléctrico.

\section{Generadores de aire caliente}

Si se quiere evitar la formación de agua condensada, disponemos de aire caliente como soporte de calor. Este sistema presenta la desventaja de precisar grandes cantidades de aire para satisfacer sólo pequeñas necesidades de calor, ya que el aire posee un calor específico bajo.

Por otra parte, en obra se precisa aire caliente para otros procesos constructivos, que no son precisamente la preparación de hormigón.

Describimos, a continuación, un aparato de fabricación alemana de fácil manejo para la producción de aire caliente (figura 10). Este puede trabajar como soplante mezclador aprovechando el $\mathrm{CO}_{2}$ de los gases de combustión para el secado en nuevos edificios, disponiendo de un rendimiento térmico elevado. Un dispositivo de entrada de aire proporciona aire caliente que sirve, además, para calentar los interiores en la zona de trabaịo. El rendimiento de calor máximo es de $80.000 \mathrm{kcal} /$ hora, pero si actúa el aparato como generador-mezclador es de $50.000 \mathrm{kcal} / \mathrm{hora}$, cuando trabaja con dispositivo de entrada de aire. El aparato es móvil. Por medio de mangueras especiales puede transportarse fácilmente el aire al lugar de trabajo.

En las obras de tipo medio casi no se encuentran instalaciones grandes para aire caliente. Actualmente se dispone de turbinas de calentamiento con un rendimiento/hora de unas $180.000 \mathrm{kcal}$ con un arrastre de aire de unos $7.000 \mathrm{~m}^{3} /$ hora (temperatura $\mathrm{t}=80^{\circ} \mathrm{C}$ hasta $200^{\circ} \mathrm{C}$ ) .
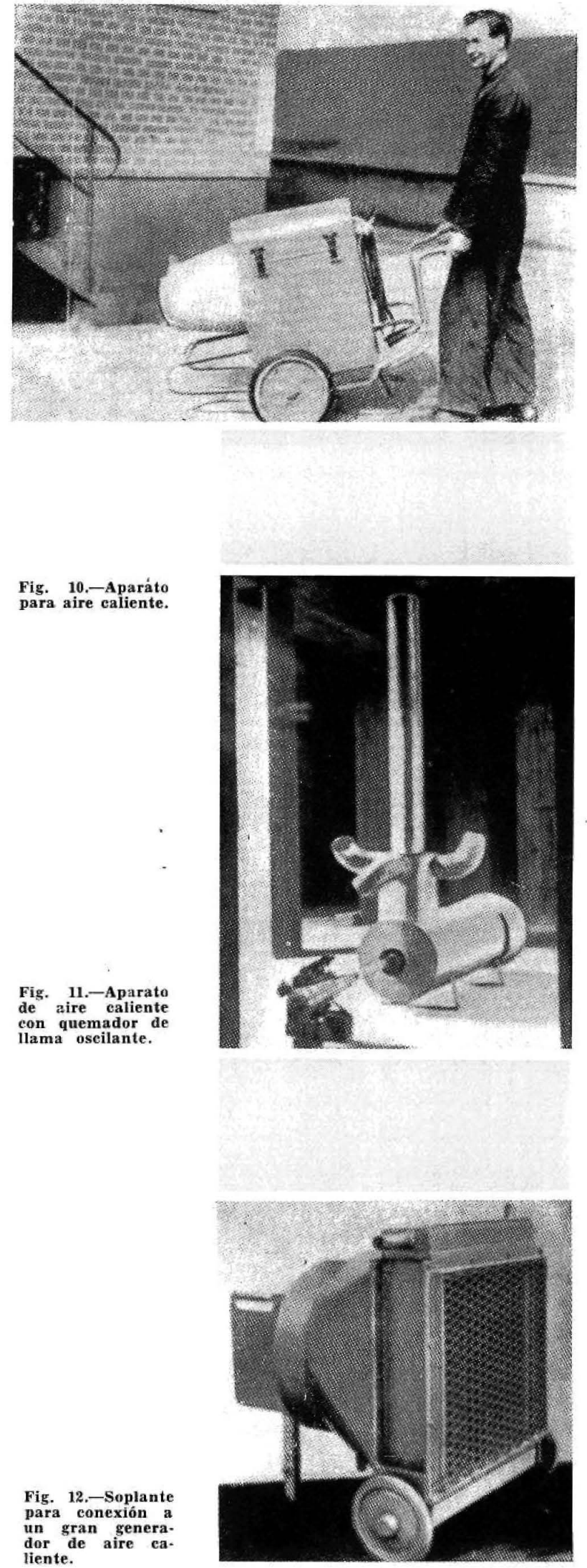


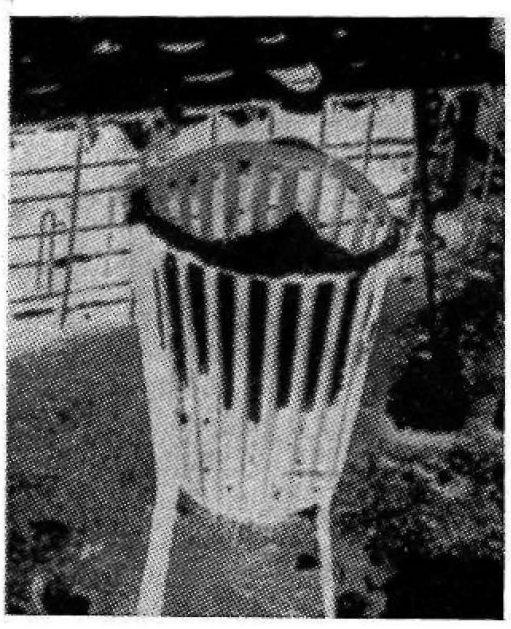

Fig. 13. - Cesta
simple de coke.

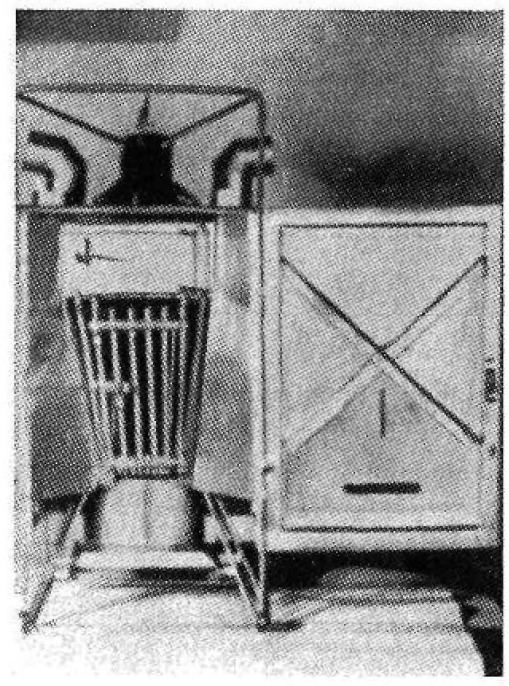

Fig. 14.-Cesta de coke para calefaccín de estancias.

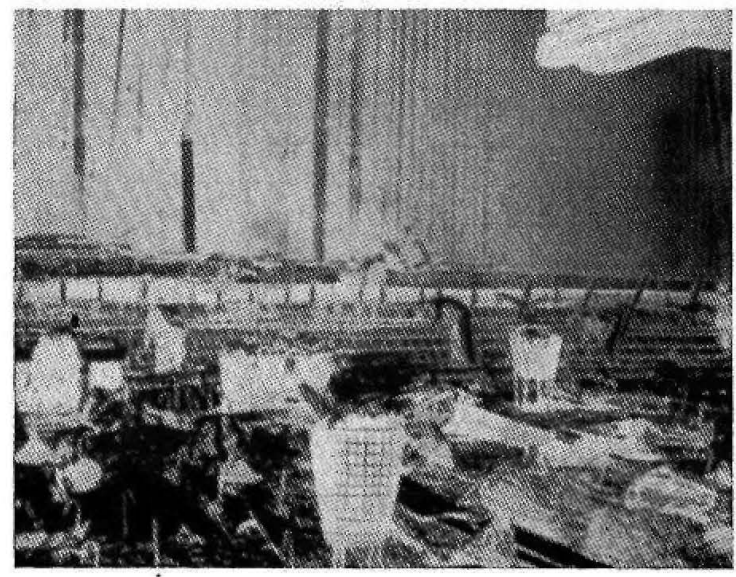

Fig. 15.-Obra importante calentada por cestas de coke.
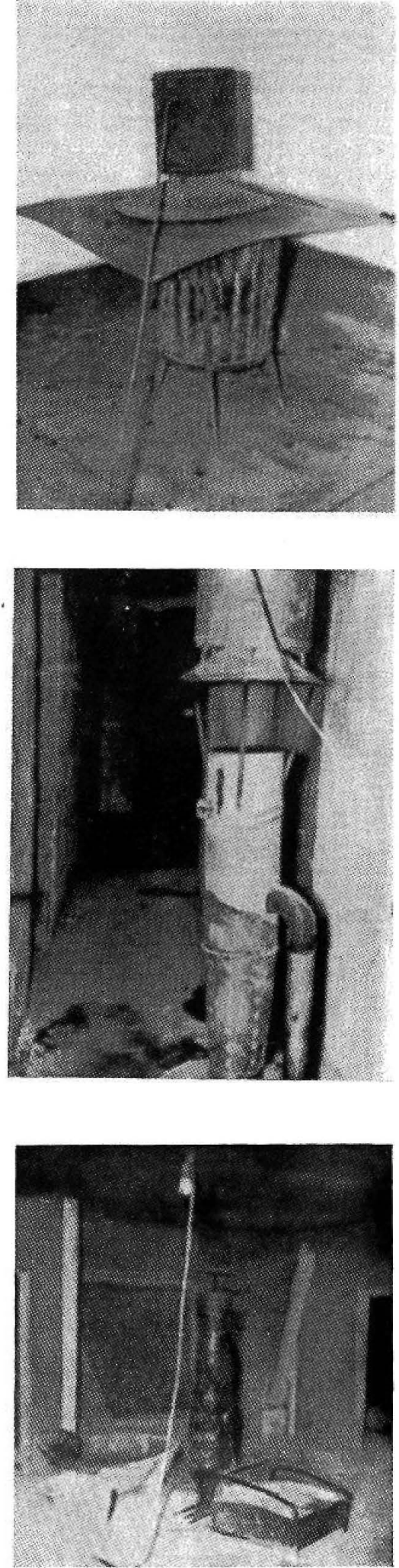

Fig. 16. Cesta de coke con soplante como aparato para aire caliente- Fig. 17. Estude secado alimentada con petróleo. 
Deseamos citar todavia un pequeño aparato para aire caliente que se alimenta con el hogar de Scwing (figura 11). Este quemador calienta en un espacio cerrado el aire absorbido por una soplante. El aire sale por 4 aberturas a los lugares de trabajo. Se calientan fácllmente $5.000 \mathrm{~m}^{3}$ de aire/hora con este generador a una temperatura de 50 a $60^{\circ} \mathrm{C}$. Este aparato trabaja casi sin conservación.

La figura 12 muestra la soplante que obtiene su aire caliente de una de las calderas antes citadas, pudiendo suministrar tanto aire caliente como agua caliente y vapor.

EL hormigón que se encuentra en su fase de endurecimiento en el encofrado debe protegerse un plazo determinado para evitar su enfriamiento y poder conseguir una cierta resistencia. Con temperaturas muy bajas no es suficiente una simple cubrición, sino que es necesario dar calor desde el exterior.

Esto se puede conseguir con la maquinaria antes descrita. Se puede conducir vapor debajo de la cubierta. Tomando ciertos cuidados se puede tomar también aire como soporte de calor. En este procedimiento debe tenerse en cuenta que el aire caliente absorbe con gran avidez el agua, la que, por otra parte, precisa el hormigón para su endurecimiento.

El método más simple y efectivo para proteger la fase del endurecimiento del hormigón en el encofrado se consigue con la disposición adecuada de estufas. Por ejemplo, al tratarse de un forjado se pueden colocar por debajo estufas durante el endurecimiento. Este tipo de estufas se puede emplear tambièn para calentar o destilar pequeñas cantidades de áricios. La estufa más simple de este tipo es el cesto de coke (figura 13), que sólo tiene la desventaja de que sus fases de combustión son molestas para los operarios que trabajan en las proximidades. Esta cesta es, además, un aparato relativamente antieconómico por radiar su calor en todos los sentióos originando, por lo tanto, elevadas pérdidas térmicas. En la actualidad se dispone de cestas de coke especialmente preparadas para la construcción (figura 14). Hasta hace pocos años la construcción de invierno en las grandes obras se realizaba casi exclusivamente con cestas de coke (figura 15). Esta cesta se puede transformar simplemente como aparato para aire caliente, instalando un ventilador (figura $\mathbf{1 6}$ ).

En los últimos años se han empleado en Alemania. con gran éxito, estufas simples de aceite, con muchos tipos, y gama amplia de rendimientos (figura 17). Estas estufas son económicas. Los tipos más corrientes suministran de $10.000 \mathrm{a} 30.000 \mathrm{kcal} / \mathrm{h}$. Sus gases de combustión no son perjudiciales. Se dejan desplazar fácilmente. Algunos de estos aparatos aparecen equipados con un pequeño recipiente para agua caliente, que sirve como elemento de calefacción para los alojamientos y para la preparación de agua caliente. Un secador sueco alimentado con petróleo puede suministrar calor regulable entre 7.000 y $25.000 \mathrm{kcal} / \mathrm{h}$. (figura 18). Su rendimiento corresponde, aproximadamente, al de 2 ó 3 cestas de coke. La conservación de estas estufas es muy simple. Sólo requieren en la práctica su encendido y la recarga del depósito de combustible. Con una carga se puede trabajar hasta durante 12 horas.

Como fuente de calor se puede emplear también gas liquido. Una constructora de Berlín fabrica por este procedimiento aparatos destinados a mantener el calor para la construcción en invierno, de los que uno suministra $10.000 \mathrm{kcal} / \mathrm{h}$., consumiendo $0,9 \mathrm{~kg}$ de gas $\mathrm{y}$ tienen un peso de $9 \mathrm{~kg}$. Otro tipo, con un rendimiento de $30.000 \mathrm{kcal}$, consume $2,8 \mathrm{~kg}$ de gas, $\mathrm{y}$ tienen un peso de $16 \mathrm{~kg}$. Este tipo de aparatos precisa muy poca conservación y resulta económico en su funcionamiento.

\section{Energfa eléctrica}

En Alemania se emplea relativamente poco la energia eléctrica como fuente de calor en invierno en las obras de tipo medio. El elevado consumo necesario, sólo se puede conducir con instalaciones especiales que originan dificultades para las obras de poco volumen.

Como calefacción para estancias, para mantener el calor y para calentar los alojamientos de los operarios se emplean ya instalaciones eléctricas, las que, en la mayorfa de los casos, se conectan directamente a la red de alumbrado disponible.

En Alemania no son corrientes todos los dispositivos necesarios para calentar el hormigón directamente con corriente eléctrica, a no ser que se dispongan elementos de resistencia en huecos o se emplee la armadura directamente como conductor.

En la República Federal se dispone de las condiciones técnieas para un calentamiento de este tipo. Este procedimiento se utiliza en Rusia, en China y, de vez en cuando, en los Países nórdicos. 

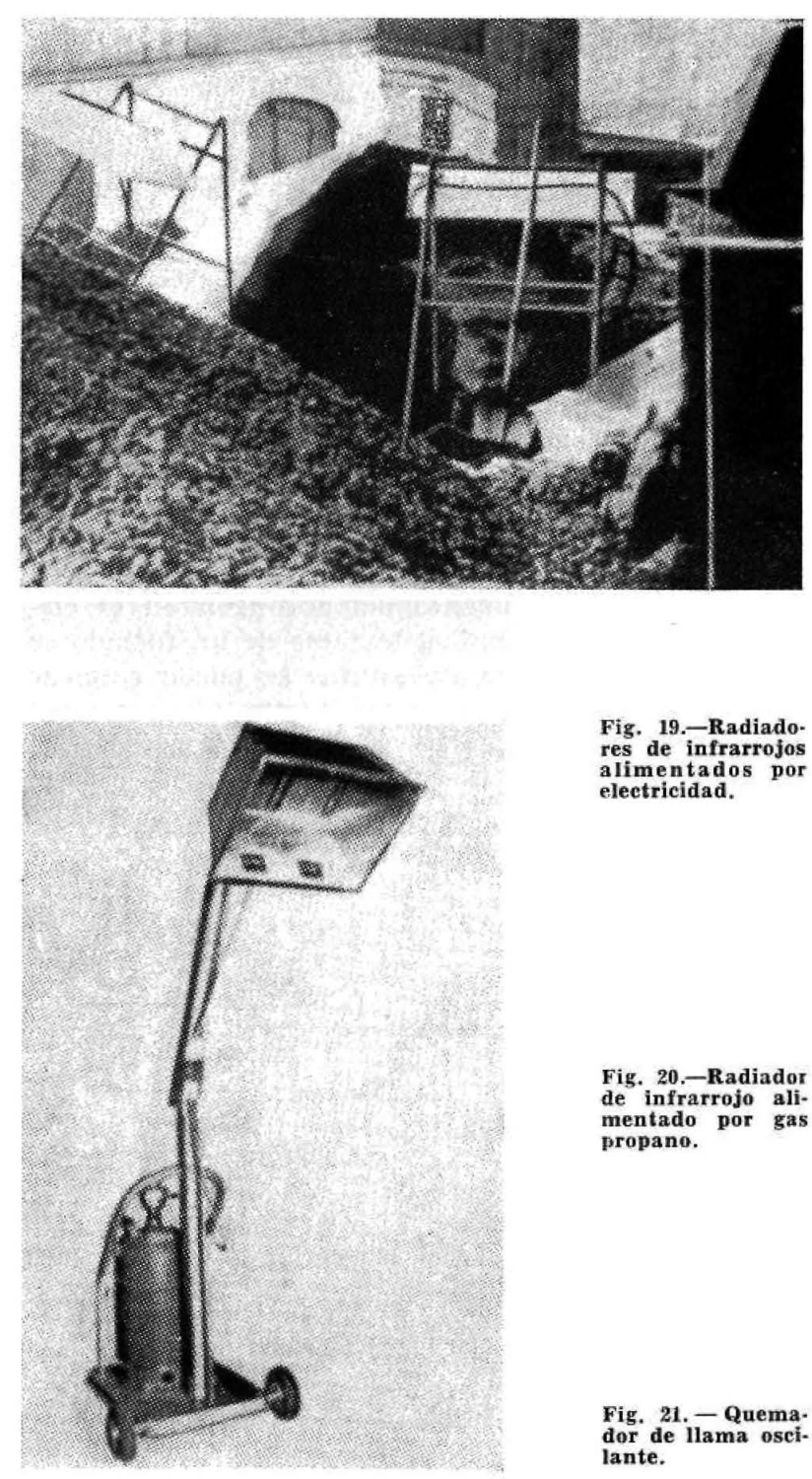

Fig. 19.-Radiadores de infrarrojos eleetricidad.

Fig. 20.-Radiador de infrarrojo alimentado por gas propano.

Fig. 21. - Quema dor de llama oseilante.

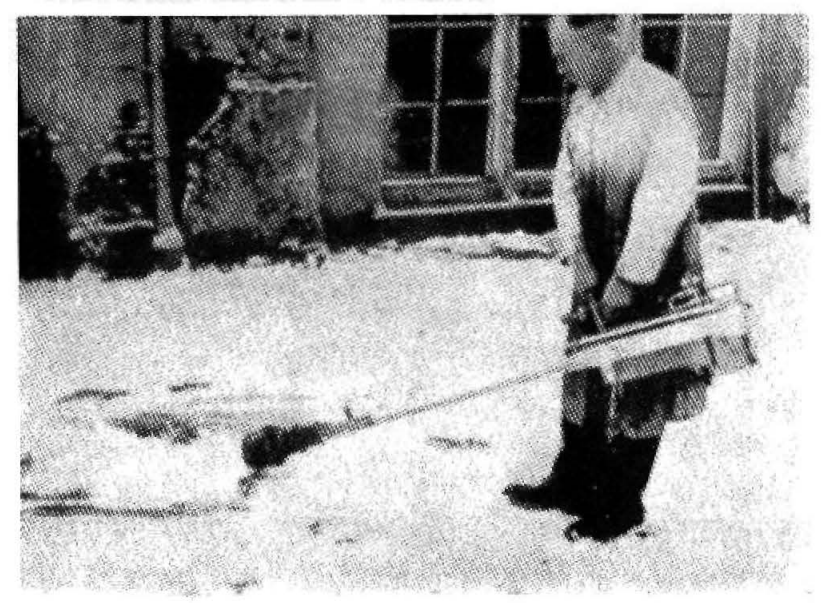

En Alemania no se usa este sistema por crear gastos muy elevados. Los demás procedimientos son más fáciles y ofrecen mayor seguridad para el trabajo en nuestras condiciones climatológicas.

\section{Radiadores de infrarrojos}

En Alemania se ha utilizado alguna vez la corriente eléctrica como fuente de calor: hace unos años se adaptaron por primera vez los radiadores de infrarrojos como fuente de calor. Estos radiadores se componen de reflectores de aluminio y radiatores de cuarzo fundido, teniendo cada uno de ellos una potencia de $3 \mathrm{ki}-$ lovatios (figura 19). Este aparato se puede conectar en corriente trifásica de $\mathbf{3 8 0}$ V. Existen también radiadores para $220 \mathrm{~V}$ alterna y $500 \mathrm{~V}$ trifásica.

La energia de estos radiadores es suficiente para solucionar pequeños problemas de calor. Por ejemplo, con un radiador de tipo grande se pueden calentar $8 \mathrm{~m}^{2}$. Los gastos de funcionamiento resultan relativamente elevados. En ciertos tipos de obras se puede soportar este gasto; por ejemplo, en una obra donde se fabrican pilares de hormigón «in situ» pueden calentarse a la vez los áridos. En una obra en la que trabajan pocos operarios especializados fue una gran ventaja el poder disponer de una fuente de calor sin gran trabajo inicial, para poder realizar los trabajos de preparación en la jornada. El tiempo de calentamiento es breve. A la vez, se calentaba la zona de trabajo, y como esta radiación es casi independiente de las corrientes de aire no se precisaban grandes cubriciones.

Para las obras se construyen radiadores de tipo lámpara, ofreciendo una elevada intensidad y un gran rendimiento por superficie en comparación con los radiadores de tubulares.

Los radiadores de infrarrojos se pueden calentar tamkién con gas propano (figura 20). Presentan las mismas ventajas que los alimentados por energia eléctrica $\mathrm{y}$, además, no precisan una instalación difícil. Estos tipos pueden desplazarse fácilmente en la obra y se pueden emplear en zonas de trabajo donde se precise un breve calentamiento. 
Como ya hemos indicado antes existe la idea en la construcción de invierno de no introducir ninguna cantidad de agua adicional, y que justamente el soporte ideal de calor, el vapor, produce agua al condensarse que puede originar dificultades en la obra.

Existe un aparato que inicialmente se habia desarrollado para un fin diferente a la construcción de invierno $\mathrm{y}$ que suministra elevadas cantidades de calor sin la adición molesta del agua. Se trata del quemador de pulsaciones, conocido como "aparato de fuego oscilante" que representa una fuente de calor de mucho rendimiento. Se puede emplear directamente o como elemento adicional a otras fuentes de calor (figura 21). El serpentín de calentamiento y el aparato de aire caliente, citados anteriormente, están equipados con un aparato de llama oscilante. Esta máquina se puede llevar fácilmente por un hombre en estado de funcionamiento. Desarrolla un rendimiento de calor graduable de 30.000 a 150.000 $\mathrm{kcal} / \mathrm{h}$. Arranca a temperaturas bajas. En el caso del aparato de llama oscilante se origina una mezcla de aire y combustible como en el caso de un motor de gasolina. Los gases originados en la explosión son lo bastante potentes para lanzar el gas combustible por el tubo largo. Por esto se forman oscilaciones en este sistema de vacio que originan una depresión que arrastra la próxima mezcla de aire y combustible. Este proceso se provoca automaticamente una vez hecho el encendido. Un aparato de fuego oscilante de este tipo se ha equipado recientemente con un intercambiador de calor. El tubo de envoltura-acoplado sobre el tubo de la llama-se puede introducir directamente en el líquido a calentar con ayuda de una brida dispuesta en el depósito. En este caso trabaja como un calentador de inmersión. Para las amasadoras de hormigón equipadas con un depósito de agua se podria emplear este procedimiento como medio efectivo y simple.

Con un dispositivo adicional similar se puede montar también una soplante de aire caliente a base del aparato de llama oscilante, que puede tener, igualmente, una aplicación muy buena en las obras. En el caso de movimientos de tierras es importante disponer de un aparato de este tipo para poner en marcha los camiones y otras máquinas con objeto de provocar el arranque del motor.

Reunidos en batería pueden realizar buenos trabajos de secado en obra (figura 22).

\section{lluminación de las obras}

Depende de su potencia, si ha de servir como: Iluminación de orientacion.

Iluminación de exteriores; 0

Iluminación interior.

Para esto se emplean los cuerpos corrientes de iluminación alimentados por corriente eléctrica o por petróleo (figura 23). Las zonas de trabajo deben disponer de una luz clara, pero sin deslumbrar. I,a buena luz no sólo mejora la calidad y el rendimiento, sino que también reduce el peligro de accidentes. Las instalaciones de iluminación deben ser fijas. Los puntos de luz no fijos provocan una molestia como consecuencia de las sombras proyectadas. Se debe considerar siempre que los operarios que ac trabajan con la Iuz del día están sometidos a una redueción de rendimiento y a un aumento de accidentes. La experiencia ha demostrado que es mejor aprovechar al máximo la luz diurna, que trabajar con luz artificial por buena que sea. En todo caso debe haber buena luz, aún de dia, en las zonas oscuras.

\section{Profección térmica}

Cada caloría que se produce con la maquinaria descrita cuesta dinero. De los gastos de primera instancia y de su funcionamiento se deduce el costo de la producción de calor por el procedimiento empleado con un tipo de maquinaria en la construcción distinto del normal. En el caso de aparatos para calor aparece un gasto inicial superior en un 60 por 100 en comparación con la restante maquinaria; este valor se debe tomar para obtener los valores reales. Se puede suponer acertada la suposición de un 20 por 100 para maquínaria de la construcción en invierno.

El mantener lo más reducido posible esta inversión es un problema de economia. Por este motivo el éxito económico de todas las máquinas de calor se debe aumentar, en parte, protegiendo la energia calorifica producida, es decir, protegiendo del enfriamiento las partes constructivas calentadas. Este proceso de trabajo de la obra en el invierno tiene una importancia considerable en relación con los resultados económicos y técnicos. La protección térmica adecuada, lo mismo que el consumo de calor, pueden fijarse por cálculo. El calor se transmite por una ley lineal simple, que es la de Fournier, al pasar del punto de más temperatura al de menos. La cantidad de calor que pasa por un material en un tiempo determinado depende, principalmente, de una propiedad especifica del material, que es su conductibilidad térmica, aparte de su espesor. Si se ha determinado la temperatura exterior y se conoce la temperatura interior que se quiere mantener (hormigón en fase de endurecimiento o protección de una hormigonera). se podrá determinar el tipo de envoltura que ofrezca una protección térmica suficiente. En la actualidad existen numerosos tipos de esterillas de lana de vidrio, lana de escoria, paja, y combinaciones de muchos materiales, cuyos coeficientes de transmisión de calor son conocidos y a base de los cuales se puede juzgar si esta protección cumple satisfactoriamente las con- 


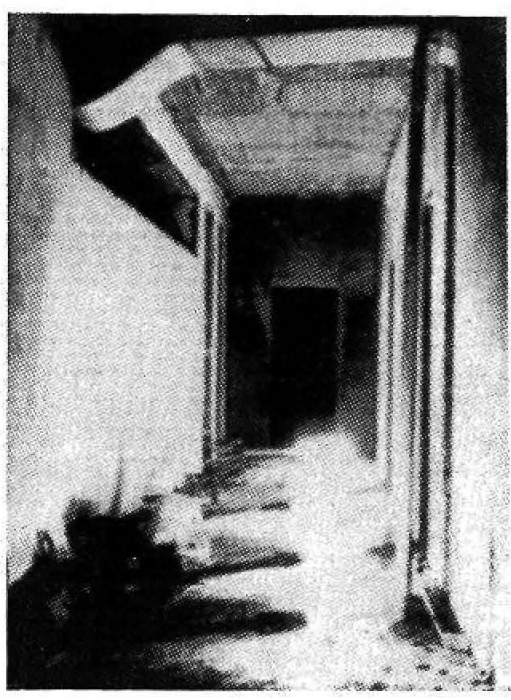
Fig. 22. - Bateria llama oseilante para secar un edificio en obra.

Fig. 23.-Proyectores eléctricos pavierno.

Fig. 24.-Armadura para envoltura de una obra de invierno con cu-
bierta de plástico.
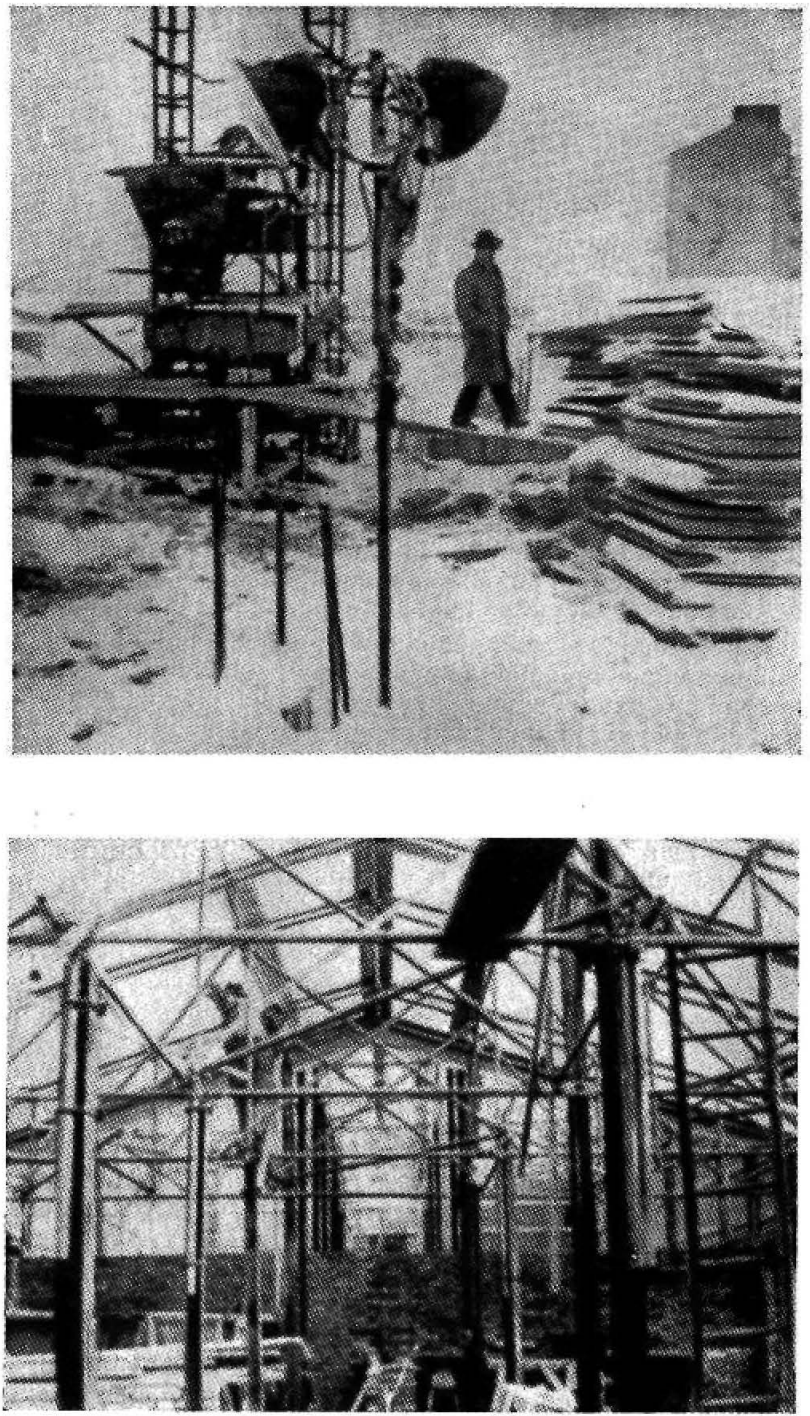

diciones para la construcción especial en invierno.

Ya el encofrado puede servir como envoltura protectora 0 , al menos, se puede prever su posible aislamiento. Para la obra de invierno se emplean, por ejemplo, encofrados dobles, con una cámara de aire intermedia que hace de aislamiento y evita, de un modo efectivo, las pérdidas de calor. También se pueden emplear otros materiales modernos esponjosos, a base de resinas artificiales (Styropor, Peresta y similares), bien directamente como encofrados o bien como capa intermedia en los encofrados de invierno.

Con frecuencia es suficiente proteger los materiales o los elementos frente a los efectos de la nieve y la lluvia. Para la cubrición en estos casos se usa todo tipo de plásticos laminares introducidos en Alemania en los últimos años. Estos materiales son en sí demasiados finos para conseguir una protección térmica efectiva, pero son suficientes para anular los efectos de las precipitaciones. Las telas de estos materiales tienen la desventaja de que no son de gran resistencia, cosa muy desfavorable en el trabajo rudo de la construcción. Para compensar esta desventaja se ha armado el material plástico.

Con esto se está en condiciones de poder cubrir grandes superficies, incluso se ha llegado a cubrir toda una construcción (figura 24). Con armaduras provisionales de madera o metal se protege la obra. Este procedimiento empleado en Rusia hace ya casi 15 años, se denomina procedimiento térmico. Es el procedimiento más seguro, pero, por el momento, también es el método más caro en la construcción de invierno. También se ha demostrado en las obras alemanas que es, naturalmente, mucho más caro que los procedimientos empleados «al descubierton.

El procedimiento de obra con una envoltura completa asegura siempre el éxito, tanto técnica como económicamente. Con esto se crean-lo que también es importante de considerar en nuestra situación actual-buenas condiciones de trabajo. Cuando sólo se pueden proteger ciertas partes de un edificio en el periodo de malas condiciones atmosféricas, sólo se tiene ocupación para una parte del personal obrero. Con esto se originan gastos que son elevados en una gran obra de mucho personal, gastos que podrian compensarse desde el punto de vista económico creando mayores inversiones. 
Fig. 25.-Nave de aire caliente. En el interior se efectúa la construcción de un nuevo edificio.

Esto rige también para la solución más elegante encontrada en este sentido. Nos referimos en este caso a la nave especial de aire caliente, que se infla y se mantiene sin armadura. Esta abarca toda la obra. Naturalmente, el coste es muy elevado. Si se aprovechan todas las posibilidades técnicas, por tratarse de una nave de muy fácil desplazamiento se pueden efectuar todos los trabajos necesarios en la obra especialmente sensibles a las condiciones atmosféricas, $\mathrm{y}$ aprovecharse en obras sucesivas de la misma envergadura una vez hecho el cerramiento. Con ello se reduce, en consecuen-

cia la inversión económica para poder convertir este elemento en una ayuda importante para la construcción en invierno. A continuación, citaremos un informe breve sobre maquinaria y aparatos de construcción que no deben faltar.

\section{Hormigón transportado para la construcción en invierno}

Gran parte del gasto adicional para la elaboración de hormigón en la obra de invierno se puede afrontar recibiendo el hormigón de una central. Naturalmente, el suministro de hormigón fresco se encuentra sometido a circunstancias comunes en la construcción en invierno y en verano. Por ejemplo, no puede transportarse más que hasta una cierta distancia y, por tanto, sólo puede emplearse en obras que estén en la ciudad. Justamente por esto se deberían aprovechar las ventajas reales que se obtienen en el hormigón transportado en el invierno. En los Países Nórdicos y, también, en Holanda y Dinamarca, es corriente solicitar a una fábrica hormigón a una determinada temperatura de colocación. En estos casos, el suministrador responde de la garantía para esta temperatura. Se ha demostrado que en el transporte en camiones normales cubjertos el enfriamiento es insignificante, incluso a bajas temperaturas y, por tanto, puede llegar a la obra con suficiente temperatura para su colocación. Es natural que acorte, en lo posible, el recorrido del camión a la obra. Por otra parte, se ha demostrado que bajo las temperaturas normales en las que todavía se trabaja en Alemania (hasta 7 ó $10^{\circ} \mathrm{C}$ bajo cero), no es necesario aislar el cubilete de transporte de la grúa o montacargas para evitar pérdidas elevadas de calor.

\section{Resumen}

Esta publicación informa sobre la maquinaria y aparatos para el hormigonado en invierno. No es posible citar completamente todos los tipos de máquinas y aparatos existentes en Alemania. En resumen, podemos decir lo siguiente:

En la República Federal Alemana se dispone de toda la maquinaria y aparatos necesarios para conseguir un éxito en la construcción de invierno, en calidad y precio tales que no significan un impedimento para la realización de la construcción bajo estas condiciones. Aunque en los últimos años se han conseguido buenos éxitos en el incremento de la construcción de invierno, queda todavia un largo camino por recorrer. Las condiciones técnicas generales están a disposición en la República Federal Alemana, especialmente en relación con el tema que acabamos de tratar. Estas condiciones se han publicado, en forma concreta, en las llamadas $«$ Directrices Técnicas para la construcción en invierno».

Fig. 26.-Construcción de viviendas en el interior de la nave de la figura 25.

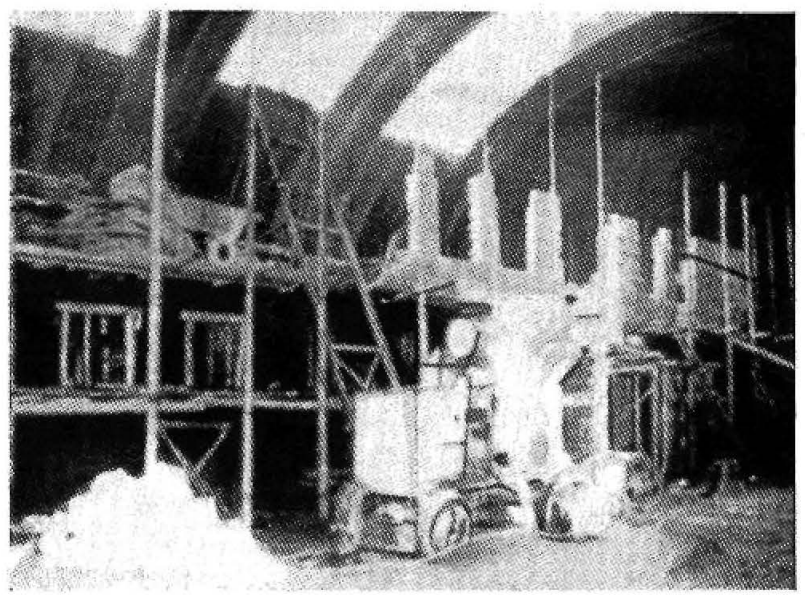

